\begin{tabular}{ccc}
\hline International Journal of Engineering \& Technology, $7(4.38)(2018) 118-121$ \\
SPC & Website www.sciencepubco.com/index.php/IJET \\
Research paper & Technology \\
\hline
\end{tabular}

\title{
Engineering Education for Forming Students' Risk-Oriented Consciousness
}

\author{
Irina G. Dolinina ${ }^{1 *}$, Lyubov K. Geykhman¹, Oksana V. Kushnaryova ${ }^{1}$, Vyacheslav I. Kazarenkov² \\ ${ }^{1}$ Perm National Research Polytechnic University, Komsomolsky prospect, 29, Perm, 614990, Russia \\ ${ }^{2}$ Academy of Engineering, Peoples' Friendship University of Russia (RUDN University), 117198, Russia, Moscow, Miklukho-Maklay \\ Street, 6 . \\ *Corresponding author E-mail: dolinina.i.g@mail.ru
}

\begin{abstract}
The paper outlines the research on educational processes aimed at forming risk-oriented consciousness in students as an essential component of life safety culture. Risk-oriented consciousness formation in students attending a technical university is advisable in view of them getting ready for future professional activities and its advisability is the core idea of all the work done. The paper contains an authors' interpretation of "risk-oriented consciousness of students attending a technical university" concept. To achieve the research goal, the authors applied a design technique. As a result, an educational technology for forming risk-oriented consciousness in students was developed. The authors also highlight obvious prospects and necessity to get further insights into the problem under consideration.
\end{abstract}

Keywords: Educational technology; Formation; Students' risk-oriented consciousness;

\section{Introduction}

To solve problems related to providing safe industrial objects functioning, the society requires professionals with developed life safety culture. They are to have a peculiar risk-oriented consciousness and to treat social, ecological, and industrial safety provision as an imperative. And such specialists need not only knowledge and skills related to safety provision, but also deeper insight into their goals and consequences of their actions for the society and the environment. It means that the most important goal of the educational process in the sphere of safety provision is to form students' consciousness based on profound understanding of the basic principle, namely unconditional priority given to safety when any personal or occupational tasks are being solved $[1,2]$ Risk-oriented consciousness interprets all the incoming information with all the knowledge and skills a person possesses, his or her value attitudes and individual experience, and helps to choose a relevant action in a situation when risk is unacceptable.

As the examined problem is complicated and ambiguous, it allowed us to detect a number of contradictions between:

-Objective demand the society has for highly qualified professionals who possess knowledge and skills for providing individual and collective safety, and an actual low level of life safety culture in students. The reasons for this are poor pre-university training, lack of motivation to obtain new knowledge which is characteristic for most students, out-of-date educational techniques which don't correspond to contemporary education system conditions, smaller work content of subjects included into natural-sciences cycle and humanitarian cycle;

-Possibilities existing in a research polytechnic university and absence of complex scientific and methodological support for riskoriented thinking formation $[3,4]$;

-Students' need to be active subjects in educational activities and their actual role as passive objects to be estimated;
-Necessity to assess forming competences and absence of a diagnostic toolset which would allow to detect difficulties related to mastering new knowledge in due time and adjust the educational process thus managing its quality.

The following principles gave the methodological grounds to the research: historism, systemacy, value orientation, teaching and education interrelation [5].

Historism made for determining attitudes which thinkers, public figures, and scientists had towards life safety culture, risk, risk analysis and risk management in the society, in industrial environment, and in education, in various historical epochs.

Systemacy determined our research methodology which allowed us to examine an educational space forming students' risk-oriented consciousness as interrelated components with all the correlations between them allowing for its integrity, hierarchical patterns, structurization, and multiplicity.

Value orientation meant forming such a system of values in students which included, among other things, human rights, private property inviolability, and pluralism.

Teaching and education interrelation allowed us to create teaching and educational components of risk-oriented consciousness formation. Educational tasks were performed as a complex impact on a student (school students, university students, students attending skills development courses) aimed at influencing his or her perception of the world, value attitudes, and personal qualities.

\section{Theoretical grounds}

Risk-oriented consciousness formation in students attending a technical university is a society goal, a state and a social demand. Risk-oriented consciousness is being formed during the whole life of a person. This research concentrates on a goal-oriented educational process aimed at changing a student's personality which 
involves adjustment of teaching and educational impacts on a student attending a technical university.

Educational space which forms students' risk-oriented consciousness functions only if there is a correlation and interaction between the following elements:

-Methodological grounds: statements on education being goaloriented, works on philosophy, sociology, a safe personality education, ecological education, axiological aspects of education, person-oriented and person-activity approaches in education, technologies implementation in teaching and education, personal responsibility, competence approach, methodological concepts of scientific-educational research;

-Educational space contents (knowledge and skills related to providing national, industrial-ecological, and personal safety);

-Created competences: cognitive, social-political, cross-cultural, communicative, creative, habitual, continuous learning;

-Categorical tools: students' risk-oriented thinking formation, students' life safety culture; ecological, industrial and occupational safety, risk analysis, avoidance, acceptance, and management:

-Created awareness: values, social goals, convictions [3];

-Individual levels of risk-oriented consciousness;

-Educational conditions making for efficiency of students' riskoriented consciousness formation (developed program and manuals).

We see formation as a complex and educationally advisable process aimed at making up of a student's personality which comprises development, education, and learning.

Future occupational activity of a graduate requires specific thinking. Obviously, an engineer needs a technical thinking but it's not enough; contemporary techno space is changing too rapidly, and an engineer also needs a risk-oriented thinking which allows to provide safety and to identify a risk in time, to assess it and to prevent hazardous situations.

We assumed in our research that risk-oriented consciousness was an individual's ability to determine whether a situation was a risky one, to identify, analyze and assess risk, to reveal regularities which caused it, and to provide human safety applying all the obtained knowledge and basing on the systemic approach. They should be able to do it even in non-standard situations when there are no ready technical solutions and ways of action.

Our developed definition is valuable because it allows to reveal inner specific methodological components and conditions for students' risk-oriented consciousness formation practices.

Risk-oriented consciousness accomplishes a number of interrelated functions [6], most significant ones being: heads.

-Cognitive function which involves awareness about existing equipment and technologies;

-Practice-transforming and practice-mobilizing function (transformation, working out new solutions to technical and technological problems);

-Educational function (specific qualities developed in each member of a society);

- Critical function (critical attitudes towards the existing reality, out-of-date stereotypes overcoming);

-Predictive function (development forecasts).

The performed analysis and our own experience allowed us to assume that it was necessary and quite sufficient to spot out three levels in risk-oriented consciousness to determine whether the educational process was efficient or not [5].

The first (low) level is an information-neutral, emotional one. I involves indifferent and nihilistic attitudes which a student has towards life safety culture, absence of knowledge on existing hazards, risks, and principles of providing personal, public, and other safety. A student's views are determined by experienced moods and under influences exerted by his or her closest circle (family and friends). Personal values system is not developed, private and personal egoism is apparent.

The second (average) level is selective-fragmentary and emotional-volitional one; it means certain information about future occupational activities is accumulated, risk concepts are being mas- tered, obtained knowledge is reproduced; this level is formed within professional training of a future specialist who is to provide life safety. Axiological values system is being formed. An individual starts to become aware of his or her duty and responsibilities but he or she still lacks a clear system of concepts and guidelines in the sphere of providing industrial, occupational, and ecological safety, risk analysis and management.

The third (high) level is a systemic-value, emotional-rational, and a creative one. Here a student has a system of universal, occupational, and individual values. A student possesses comprehensive professional knowledge and applies it freely. He or she has a personal awareness how significant his or her actions are for solving state or social tasks.

\section{Educational technology}

All paragraphs must be justified alignment. With justified alignment, both sides of the paragraph are straight. We performed theoretical research on philosophical and educational aspects of riskoriented consciousness formation simultaneously with designing an educational technology aimed at students' risk-oriented thinking formation

We applied a technological approach in our research as "...giving grounds for conceptual and projecting mastering of various social activity spheres and aspects, it allows to analyze and systematize practical experience and its implementation on the scientific foundation; to find complex solutions to social and social-educational problems; to create favorable conditions for a person development; to lower impacts exerted by unfavorable circumstances; to apply all the available resources in a most optimal way; to choose most efficient and developed new technologies to solve social problems" [7].

Authors applied the following educational technologies features described by V.N. Voronin in their creative research: "Contemporary educational technologies, as opposed to conventional ones, have a number of distinctive features: clear goal-setting, a result which can be diagnosed, both teachers' and students' activities can be given an algorithm, tutorials can be designed, education quality can be managed, repeated, adjusted, and guaranteed, and educational process is systemic and economically efficient" $[8,9]$.

An educational technology aimed at students' risk-oriented consciousness formation is understood in this research as a design and reputability of educational actions aimed at achieving a professional education goal which is to form students' risk-oriented consciousness. The result of it is acquired knowledge on social, ecologic, and techno risk, readiness to prevent hazardous situations on the basis of value orientation at providing people's safety [10].

An educational technology aimed at students' risk-oriented consciousness formation includes an informative and a procedural component [11].

The informative component is implemented via content modules ("Risk identification", "Risk assessment", and "Risk factors prediction and management"), via inter-subject correlations, via analysis and observation over real life and industrial situations involving risks. Risk-oriented consciousness as a concept and its basic characteristics are formed; students learn how theoretical grounds of "risk-oriented consciousness" concept appeared and developed; risk-oriented consciousness structure and its basic features are determined. Risk assessment and risk management procedures are studied, and students learn how to identify and to assess risks. A special attention is paid to social and technical responsibility in assessment procedures and managerial decision-making aimed at managing risks when learning tasks are solved; to stages in risk analysis (identification, assessment, and lowering); to working out strategies in risky situations [6].

Risk-oriented students' consciousness is formed via various educational, learning, and developing techniques comprised by the procedural component. 
Organizational techniques are visualized (movies, presentations, and technical experiments). It includes problem lectures, practical studies with scientific research elements, projects development, essays and analytical reports writing, risk-related cases analysis, diversion analysis, brainstorms, and business games.

Various organizational forms of learning process are applied depending on employed techniques. It can be individual, pair, group. front-end, or territorial work.

Diagnostics is implemented through examining educational results (knowledge control, surveillance over skills development, reflection via questioning). Diagnostics (diagnostikos in Greek, meaning "able to recognize") means determining peculiarities which educational process results have: acquired knowledge strength, skills development level, and ability to realize oneself within an educational process. Within our research context, diagnostics is examination of students' personalities aimed at determining individual level of their risk-oriented consciousness development.

A diagnostic procedure aimed at determining individual level of risk-oriented consciousness development consists of three stages: diagnostic data collection, the obtained data treatment, conclusions and, in case of necessity, recommendations.

The obtained data allow to draw up a qualitative characteristics of risk-oriented consciousness development for a student or a group of students, to assess efficiency, and, in case of necessity, to determine most optimal educational and learning forms and techniques within the applied educational technology frameworks.

We performed the diagnostics with tests and self-control questions which we developed. But group communication has the same significance. Students come to realize themselves that "reflection is an analysis of all the learning activities aimed at their understanding, improving, and further planning; it stimulates motivation to take active part in the process during lectures and additional self-training".

Self-control and self-assessment of students' educational activities are the most significant features of a developed self-sufficient personality able to adequately assess an existing situation in his or her future professional activities, to make decisions, and to bear full responsibility for them.

\section{Implementation}

Efficiency of an educational technology aimed at students' riskoriented consciousness formation depends on its implementation. Conditions which are necessary for its successful implementation are created by tutorial sets on "Working conditions examination" and "Working conditions provision systems" subjects which are manuals providing problem solution process integrity. They were developed to find such forms or techniques which could make for achieving optimal results under specific conditions or circumstances.

Learning materials of "Working conditions examination" and "Working conditions provision systems" subjects are divided into three modules: Risk identification, Risk assessment, Risk factors prediction and management.

The first two modules are parts of "Working conditions expertise" subject, and "Risk factors prediction and management" module is implemented during two terms when "Working conditions provision systems" subject is studied. Subjects content remains the same; the developed technology changes educational process structure and introduces necessary theoretical and practical learning materials on risk identification, assessment, prediction, and management in the industrial safety sphere. It involves active learning techniques thus making the educational process more active, applies a design technique in a form of a "route" task which is accomplished during three terms when "Working conditions examination" and "Working conditions provision systems" subjects are being studied.

Thus "route" task topic coincides with tests topics and later on it evolves into a graduation work.
"Route" work, first of all, it has an applied nature as it involves solving quasi-occupational tasks and it helps to increase motivation when studying the subject.

Secondly, as this 'route" task is being accomplished during three terms, it allows to provide comprehensive work-up of a graduation work subject in terms of providing ecological and industrial safety and hazardous and emergency situations prevention.

"Risk identification" module is aimed at educating and developing such a feature as an ability to understand whether a situation is risky or not. It means knowledge ceases to be formal and becomes actual via "creative participation" of each individual. An ability to see a problem and to understand a situation is critical occurs when a person is able to identify a risk basing on critical and systemic analysis. It becomes possible in the educational process when business games or active learning techniques are applied, such as case study or an object diversion analysis. It allows both to enhance a student's cognitive sphere and to enrich his or her personal experience and make the subject interesting for him or her.

"Risk assessment" module includes examining and determining hazardous events possibility and gravity of their possible consequences for the environment, people, and their property. "Risk assessment" module solves the following tasks: mastering skills of obtaining objective information about an object (equipment or a working place) safety, data on the greatest object weaknesses in terms of its safety.

Assessment of (incident) risks at an industrial object is an integral part of any industrial safety management system and labor protection system at modern enterprises; it allows to apply the acquired data for predicting and managing risk factors, that is, for preventing any probable hazardous events.

Risk assessment is regulated by the following documents: Declaration on industrial safety, Safety justification, relevant sections in construction and re-construction project documentation, documentation on re-equipment, conservation, and dismantling, activity plans for localization and elimination of probable industrial emergencies consequences, results of specialized working conditions assessment.

While studying this module, students accomplish obligatory tasks applying all the above-mentioned documents issued at existing enterprises.

"Risk factors prediction and management" is an applied module and a final learning component of the educational technology aimed at risk-oriented consciousness formation. It is based on knowledge and skills obtained during studying "Risk identification" and 'Risk assessment" modules; this knowledge and skills are applied for working out well-grounded recommendations how to lower and to predict risk, and relevant managerial decisions.

"Risk factors prediction and management" module is diagnosed via tests which had a long-term approbation when students' readiness to identify risks was checked. Such tests should provide not only diagnostics of a student's thesaurus or an expert's knowledge volume, but also his or her ability to apply this knowledge to solve a specific task. For example, it can be an ability to make up a program which comprises activities aimed at preventing emergencies at a specific industrial object. Activities should be implemented and in what order, to draw up a plan of equipment maintenance repair, to draw up a schedule of instructions for personnel at their working places.

Students' abilities to apply all the acquired knowledge, techniques and tools, to solve practical tasks, and their mastering of safe labor skills and prospective analysis skills will help to prevent any situation which is hazardous in terms of occupational, industrial, or ecological safety, and to find non-standard solutions in rapidly changing conditions. While decision-making in an emergency situation is a deeply individual process, it involves creative and self-made choice on techniques, ways, and models of activities which can be both conventional and original ones as they are influenced by a person's experience. The more techniques and ways how to solve a specific task are known to a student, the more crea- 
tive he or she is in applying them in his or her professional activities.

\section{Conclusion}

To perform our research in Perm national research polytechnic university, we selected groups of students who attended "Technosphere safety" course, their training profile being "Technological processes and productions safety". They studied "Working conditions examination" and "Working conditions provision systems" subjects.

More than 200 students took part in our experiment. The experiment was performed during 5 years.

We checked all the obtained experiment results statistically to prove our assumption that teaching with the developed educational technology forms risk-oriented consciousness and, accordingly, improves quality of a specialist professional training within life safety provision system.

The obtained values of sample average parameters allowed us to assume that teaching with this newly developed educational technology resulted in better specialists' training quality and riskoriented consciousness formation in them; that is, professional risk-oriented consciousness rose from its low, information-neutral, emotional level to an average (selective-fragmentary and emotional-volitional) one and, in some cases, to its high level (systemintegral, emotional-rational, creative one).

Dynamics of changes in average values of risk-oriented consciousness structural components was insignificant in the reference groups, Student's criterion was lower than 1.64. Consequently, a zero assumption was correct for the reference groups, and discrepancies in average values were accidental.

Analysis of research results obtained in the experimental groups revealed that "Risk identification" level increased from 0.27 to 0.897. "Risk assessment" level, from 0.333 to 0.794 . "Risk prediction and prevention", from 0.372 to 0.800 . Significant growth in average values of the qualities being formed was detected in all the samplings. It was achieved due to targeted impacts exerted by the educational technology implementation into risk-oriented thinking formation.

We detected positive dynamics in our experimental groups which is within qualitative technologies range in terms of controlled parameters of an educational technology quality.

Further prospects and better insight into the problem being considered can be related to research on ways and techniques aimed at adapting and integrating the designed educational technology for students' risk-oriented consciousness formation as life safety culture component into an educational environment of any university (both educational process and off-studies work). In can be applied in designing new federal standards providing people's safety, in working out a rational component of education informative contents, in improving management techniques for managing students' education and learning.

We predict positive results which can be achieved in improving safety regulations, innovative solutions creation and implementation in the safety provision sphere, and in workers' skills development.

\section{References}

[1] Belov SV, Devisilov VA (2005), Russian concept of continuous multi-level education in the life safety sphere. Life Safety 14, 4-9.

[2] State Standard P ISO 9001-2015. "The Russian Federation National Standard. Quality management systems. Requirements" (approved by the Rosstandart order issued on September 28, 2015 N 1391-st).

[3] Dolinina IG, Kushnaryeva OV (2015), Life safety: state of an educational environment which forms students' occupational culture. Higher Education Today 9, 83-85.

[4] Dolinina IG, Kushnaryeva OV (2015), Students' life safety culture formation: a role played by a supervisor's educational activities. Contemporary Issues of Science and Education, 4.
[5] Dolinina IG, Kushnaryeva OV (2016), Forming occupational safety culture on the basis of development of students' risk-focused intellection. International Journal of Environmental \& Science Education 11(14), 6322-6334.

[6] Dolinina IG, Kushnaryeva OV (2016), Students' risk-oriented thinking actuality, definition, and operations. Contemporary Science Intensive Technologies, 9-3, 495-499.

[7] Dolinina IG, Kushnaryeva OV (2017), Pedagogical technology for forming rick-oriented thinking of the training. Humanization of Education 4, 85-91.

[8] Brushlinskiy AV, A subject: thinking, learning, imagination. Moscow-Voronezh: Izdatel'stvo instituta prakticheskoy psikhologii, MODEK 1996.

[9] Brushlinskiy AV, Volovikova MI. Psychology of an individual and a group subject. Moscow: PERSE, 2002.

[10] Kushnaryeva OV (2015), Increase of health-safety culture of trained at polytechnic higher educational institution. Modern European Researches 5, 77-79.

[11] Dolinina IG, Kushnaryeva OV (2015), Model for forming students' life safety culture in a polytechnic university. Fundamental Research 7 . 